\title{
A computational reconstruction of Papio phylogeny using Alu insertion polymorphisms
}

\author{
Vallmer E. Jordan', Jerilyn A. Walker', Thomas O. Beckstrom', Cody J. Steely', Cullen L. McDaniel', \\ Corey P. St. Romain ${ }^{1}$, The Baboon Genome Analysis Consortium, Kim C. Worley ${ }^{2,3}$, Jane Phillips-Conroy ${ }^{4}$, \\ Clifford J. Jolly ${ }^{5}$, Jeffrey Rogers ${ }^{2,3}$, Miriam K. Konkel ${ }^{1,6}$ and Mark A. Batzer ${ }^{1 *}$ (i)
}

\begin{abstract}
Background: Since the completion of the human genome project, the diversity of genome sequencing data produced for non-human primates has increased exponentially. Papio baboons are well-established biological models for studying human biology and evolution. Despite substantial interest in the evolution of Papio, the systematics of these species has been widely debated, and the evolutionary history of Papio diversity is not fully understood. Alu elements are primate-specific transposable elements with a well-documented mutation/insertion mechanism and the capacity for resolving controversial phylogenetic relationships. In this study, we conducted a whole genome analysis of Alu insertion polymorphisms unique to the Papio lineage. To complete these analyses, we created a computational algorithm to identify novel Alu insertions in next-generation sequencing data.

Results: We identified 187,379 Alu insertions present in the Papio lineage, yet absent from M. mulatta [Mmul8.0.1]. These elements were characterized using genomic data sequenced from a panel of twelve Papio baboons: two from each of the six extant Papio species. These data were used to construct a whole genome Alu-based phylogeny of Papio baboons. The resulting cladogram fully-resolved relationships within Papio.

Conclusions: These data represent the most comprehensive Alu-based phylogenetic reconstruction reported to date. In addition, this study produces the first fully resolved Alu-based phylogeny of Papio baboons.
\end{abstract}

Keywords: Alu, Retrotransposon, Phylogeny, Primates, Taxonomy, Evolutionary genetics, Papio, Hybridization

\section{Background}

The burgeoning diversity and availability of whole genome sequencing (WGS) data offers intriguing possibilities for the field of comparative primate genomics. Currently, WGS data are publicly available for over 100 primate species (NCBI Resource Coordinators 2016). Traditionally, significant interest in the genetics of non-human primates stems from their sustained role as popular research models for studying human biology and evolution [1-5]. One such primate-well established as a model for human genetics and disease susceptibility-is the Papio baboon [6-11]. In addition to close genetic relatedness, the temporal and

\footnotetext{
* Correspondence: mbatzer@lsu.edu

'Department of Biological Sciences, Louisiana State University, 202 Life

Sciences Building, Baton Rouge, LA 70803, USA

Full list of author information is available at the end of the article
}

ecological landscape of early Papio evolution bears striking resemblance to that of early hominins [2, 12-14]. Both include ancient episodes of admixture, as well as migration out of Africa into the Arabian Peninsula during the Pleistocene [2, 15-20]. Appropriately, Papio baboons represent an intriguing model for human evolution.

Papio baboons occupy the largest geographical distribution of any non-human primate genus on the African continent [21-23]. These ground dwelling Old World monkeys inhabit most of sub-Saharan Africa, to the exclusion of the tropical rainforests of West Africa and the Congo Basin, and also extend into the south-western region of the Arabian Peninsula [24, 25]. Papio systematics have been extensively studied over the past 60 years with much debate as to which forms warrant species status [25]. The disagreement is in essence philosophical, 
centered on the question of what constitutes a species. However, recent studies employ a phylogenetic species concept [26-29], positing that consistent differences in physical appearance, ecology and social behavior justify the recognition of six extant species: $P$. anubis, $P$. hamadryas, P. papio, P. cynocephalus, P. ursinus and P. kindae. In this study, we recognize all six as species.

Despite considerable interest in Papio systematics, a fully resolved consensus phylogeny remains undetermined [20, 26, 30]. Interfertility has been documented between all neighboring species, with persisting natural hybrid zones in several regions where distinct morphotypes (species) come into contact [27, 31-36]. Thus, discordance between mitochondrial, morphological, and nuclear phylogenetic reconstructions could in part stem from a dense history of admixture and reticulation persisting throughout the course of Papio evolution. Mitochondrial based phylogenies support the divergence of Papio into northern and southern lineages $[26,30]$. Individuals belonging to $P$. anubis, $P$. papio and $P$. hamadryas are consistently placed within the northern clade; with individuals belonging to $P$. kindae and $P$. ursinus comprising the southern clade. In these analyses, however, the placement of $P$. cynocephalus remains unclear with individuals found in both clades. In addition, such reconstructions have proven unsuccessful at resolving phylogenetic relationships within each clade. Thus additional analyses employing novel methodologies could further serve to elucidate evolutionary relationship within Papio.

$A l u$ elements are well-established DNA markers for the study of systematic and population genetic relationships [37-47]. In part, they are effective evolutionary characters because of their high copy number in primate genomes and sustained mobilization throughout the course of primate evolution ( $65 \mathrm{MY}$ ) [48-50]. Over 1.2 million copies have been identified in the human genome [51], with similar numbers reported for all other haplorrhine genomes sequenced to date [52-55]. Alu elements are discrete primate-specific DNA sequences $(\sim 300 \mathrm{bp})$ belonging to a class of non-LTR (long terminal repeat) retrotransposons termed short interspersed elements (SINEs). Following the transcription of a SINE, the mRNA sequence can be reverse transcribed into DNA, producing a new copy at a novel position in the host genome [56-58]. Over time, this process known as target primed reverse transcription (TPRT) can exponentially increase the retrotransposon content of a host genome. Alu elements, as well as all other SINEs, lack the requisite enzymatic machinery for TPRT; thus they require proteins encoded by larger retrotransposons known as LINEs (long interspersed elements) [48, 59, 60].

SINEs are valuable evolutionary characters because they can be assumed to be identical by descent, meaning that insertions shared between individuals were inherited from a common ancestor, rather than acquired by independent events [61]. Additionally, retrotransposons have known directionality [62, 63], with the ancestral state being the absence of the insertion. Alu elements are popular retrotransposon markers because their short length makes them particularly easy to assay using standard PCR. Considered nearly homoplasy-free [48, 49], most potential sources of homoplasy involving Alu elements can be resolved through Sanger sequencing [41, 42, 61]. Recent studies demonstrate the utility of Alu elements for Papio species identification, as well as retrieving population structure within distinct Papio species [28, 29]. Furthermore, Alu elements have been successfully used to resolve controversial relationships between primates $[38,39,42$, 64]. However, little is known about the efficacy of Alu elements to resolve phylogenetic relationships involving high levels of admixture.

Although a high-quality reference assembly currently exists for only one Papio species (P. anubis), WGS data have been generated for individuals representing all six Papio species through the Baboon Genome Consortium. Thus it is possible to conduct a comprehensive whole genome analysis of Papio phylogeny using Alu polymorphisms between species of the genus. For the present study, we created a computational pipeline to identify and characterize recently integrated Alu elements polymorphic within the genus Papio. These Alu insertion polymorphisms were used to reconstruct phylogenetic relationships within Papio. By utilizing M. mulatta as our reference, our approach placed equal evolutionary distance between each Papio diversity sample and the reference assembly [Mmul8.0.1]. The computational analyses performed in this study generated a well-supported phylogeny of Papio baboons and represents the most comprehensive Alu-based phylogenetic analysis reported to date. In addition, we report a novel approach to admixture and reticulation analysis using $A l u$ insertions.

\section{Methods \\ Samples}

Whole-genome sequencing was performed by the Baylor College of Medicine Human Genome Sequencing Center on a panel of fifteen Papio baboons: four P. anubis, two $P$. papio, two $P$. hamadryas, three $P$. kindae, two $P$. cynocephalus, and two $P$. ursinus. In order to sample an equal number of individuals from each species, we used two individuals from each of the six extant Papio species (we randomly selected two individuals from $P$. anubis and $P$. kindae) to conduct our computational analysis. Lastly, our panel included WGS data from the macaque sample used to build the latest M. mulatta assembly [Mmul8.0.1] (Additional file 1).

WGS data were accessed from the NCBI-SRA database [65]. The SRA-toolkit (fastq-dump utility) [66] was used to download paired-end next generation sequencing reads 
and convert them from .sra files to interleaved fastq files. We then used nesoni (https://github.com/VictorianBioinformatics-Consortium/nesoni; last accessed March 2018) to prune all known adapters, cleave bases with a phred quality score of 10 or lower, and exclude reads shorter than 24 base pairs in length. Two output fastq files were produced: one containing clean paired-end reads (both reads passed the nesoni filter), and a second containing unpaired orphan reads (one of the paired-end reads was excised).

\section{Polymorphic Alu insertion detection}

We developed a computational pipeline to identify and characterize recently integrated $A l u$ elements in paired-end next-generation sequencing (NGS) data. Our approach targeted young $A l u$ insertions still polymorphic within the panel of individuals listed in the previous section. The approximate chromosomal position of each candidate insertion was estimated using a split-read method (Fig. 1). The resulting genotypes, generated for all individuals in our panel, revealed markers that provided phylogenetic signal.

The AluY subfamily has been identified as youngest and most active Alu subfamily in Simiiformes [48, 67-69]. Thus, in the alignment phase, we used BWA mem [70] to map paired-end NGS reads to a consensus AluY sequence obtained from Repbase [71]. Individual reads were required to map to either the head ( $\left.5^{\prime}\right)$ or tail $\left(3^{\prime}\right)$ of the AluY consensus sequence. In addition, reads mapping to the head of an $A l u$ insertion were required to contain at least $15 \mathrm{bp}$ of unmapped/non-Alu sequence directly upstream of the (5')

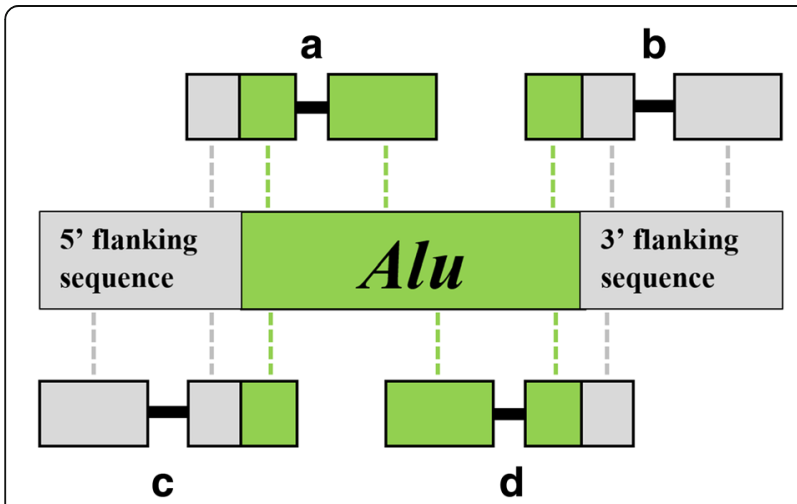

Fig. 1 Computational detection of Alu insertion polymorphisms using split-reads. Alu insertions were identified using sequencing reads spanning the Alu integration locus whether these split-reads spanned the 5' (a and $\mathbf{c}$ ) or the $3^{\prime}$ ( $\mathbf{b}$ and $\mathbf{d}$ ) end of the insertion. The four split-reads represented in this figure are labeled $\mathbf{a}, \mathbf{b}, \mathbf{c}$ and $\mathbf{d}$. Green boxes represent Alu sequence; gray boxes denote flanking sequence. If the split-read is paired and its read-pair mapped to the flanking sequence ( $\mathbf{b}$ and $\mathbf{c}$ ), these mapping coordinates were used to provide additional support for the location predicted by the split-read. If the split-read's read-pair mapped to the Alu (a and $\mathbf{d}$ ), this was used to provide additional support for the presence of the predicted Alu insertion start of the Alu sequence. Likewise, reads mapping to the tail of the consensus $A l u$ sequence were required to contain no less than 15 bases of unmapped sequence directly flanking the ( $\left.3^{\prime}\right)$ end of the sequence. Reads were mapped to the AluY consensus twice: once using the standard BWA mem parameters, and a second time using more liberal parameters (described in Additional file 2). Split-reads identified using standard parameters were later used to predict the location of an Alu integration site, while those identified during the liberal run were used simply to provide additional support for the insertion event. The Alu portion of each candidate split-read was then cleaved and remaining sequence aligned to Mmul8.0.1 using bowtie2 [72]. Splitreads were categorized as sequences that mapped uniquely to the AluY consensus and the Mmul8.0.1 assembly.

The approximate genomic position of each candidate insertion was calculated directly from the mapping positions of split-reads to Mmul8.0.1 and the AluY consensus. Alu insertion orientation was inferred from the alignment orientation of the supporting reads when mapped to the AluY consensus and Mmul8.0.1 assembly. During this phase the integration orientation of each candidate insertion was predicted in the forward orientation if positioned $5^{\prime}$ to $3^{\prime}$ on the sense strand, and the reverse orientation if positioned $5^{\prime}$ to $3^{\prime}$ on the anti-sense strand. If a split-read mapped in the same orientation to the consensus AluY and the Mmul8.0.1 assembly, it was predicted in the forward orientation. If the alignment orientations were discordant, the insertion was predicted in the reverse orientation.

Approximate genomic positions for non-reference (absent in Mmul8.0.1) Alu insertions, predicted in any of the 12 Papio individuals, were concatenated into a comprehensive list with the goal of identifying phylogenetically informative markers. All of these insertions were predicted from split-reads obtained during the standard Alu alignment run. In principle, phylogenetically informative $A l u$ elements would have integrated into the Papio lineage following its divergence from Macaca. Thus, insertions shared between Papio and the Macaca mulatta sample were excluded. Likewise Alu elements identified in only one Papio sample were phylogenetically-uninformative, and thus were also excluded from this portion of the study. The remaining loci were genotyped in every individual on the panel. The three possible genotypes homozygous present, homozygous absent, and heterozygous - were determined by analyzing sequences spanning the insertion locus. It was initially assumed that an individual was homozygous present for every insertion predicted in that sample. Likewise, it was initially assumed that an individual was homozygous absent for every locus not predicted in that individual. Insertions initially determined to be homozygous present were then re-evaluated to determine if they were in fact heterozygous present. Heterozygosity was determined by evaluating reads that 
mapped uniquely to the Mmul8.0.1 assembly. An insertion was reclassified as heterozygous if we identified reads in that individual that mapped continuously (without interruption) through the homologous empty site in the Mmul8.0.1 assembly. This empty site was defined as a sequence containing at least $15 \mathrm{bp}$ of flanking both upstream and downstream from the predicted insertion locus. Additionally, if a homozygous absent genotype was predicted in a region with a local read-depth less than two standard deviations from the global mean, the genotype was instead considered unknown.

\section{PCR validation}

The performance of the algorithm used in this study was assessed by comparing PCR validations performed for 494 loci in a panel of six Papio baboons: one from each extant Papio species [29]. From this dataset, our algorithm correctly predicted $98 \%$ of the PCR-validated events for presence/absence. In addition, the correct genotype (homozygous present, homozygous absent, or heterozygous) was computationally predicted for $93 \%$ of all events.

\section{Basal divergence analysis}

Previous phylogenetic analyses support the ancestral divergence of Papio into two clades: northern and southern lineages $[26,30]$. To evaluate this hypothesis we created a computational method to identify the basal divergence model best supported by our Papio dataset. A genus comprised of six species with three different possible phylogenetic topologies generates 31 different unique models for estimating the basal divergence (Additional file 3). For each model we determined the total number of insertions that supported and conflicted with each basal divergence. We calculated the standard deviation and z-score for each model. The model with the highest $\mathrm{z}$-score represents the basal divergence model best supported by the dataset.

\section{Phylogenetic analysis}

We used the model representing the basal divergence with the highest z-score (described in the previous section) as a pre-condition for our phylogenetic analysis. A comprehensive list of $A l u$ insertions supporting this model (consistent with the north-south split hypothesis) were used to further resolve phylogenetic relationships within Papio. A heuristic search was performed using PAUP* 4.0b10 [73]. Since it is assumed that the absence of an Alu insertion is the ancestral state of each locus, Dollo's law of irreversibility [74] was used in the analysis. Thirteen individuals were evaluated in this analysis: 12 Papio baboons, two representing each of the six extant Papio species, along with the M. mulatta sample used to build the Mmul8.0.1 assembly. Each individual received a score for each locus based on its computationally derived genotype. The presence of an insertion was scored as " 1 " for a filled site and
"0" for an empty site; unknown genotypes were scored as "?". Using PAUP we conducted a heuristic search using genotype data from Alu polymorphisms concordant with the north-south split with $M$. mulatta set as the outgroup. All loci were classified as individual insertions and set to Dollo.up for parsimony analysis as described previously [41]. Ten thousand bootstrap replicates were performed with the maximum tree space set to all possible trees.

We wrote a series of Python scripts to sort Alu insertions into clusters based on which baboons shared the insertion. This allowed us to determine the total number of $A l u$ insertions shared between different sets/combinations of baboons. Each cluster contained $A l u$ insertions shared among a distinct combination of baboons, yet absent from all other samples. For example, one cluster contained all Alu insertions shared between the P. cynocephalus samples and the $P$. kindae samples, yet absent from all remaining samples. Another cluster was comprised of Alu insertions shared between all six northern baboons, yet absent from all six southern baboons. Each cluster represents the total number of insertions shared uniquely between a particular "combination/set" of baboons. The resulting clusters were then analyzed to identify patterns of shared Alu polymorphisms. Using this script we quantified the total number of Papio indicative $A l u$-insertions, markers present in all six extant Papio species, yet absent from the $M$. mulatta sample. Clade indicative Alu polymorphisms were defined as insertions present in every species belonging to one clade, yet absent from all individuals in the other clade. In addition, we evaluated patterns of shared Alu polymorphism exhibited within each clade. In this analysis, we identified $A l u$ polymorphisms exclusive to either the northern or southern clade, yet not present in all species within that clade. Lastly, we quantified the total number of species indicative $A l u$ elements, defined as Alu polymorphisms present in both individuals belonging to a species, yet absent from all other Papio individuals in our panel.

\section{Results}

\section{Polymorphic Alu identification}

WGS data for multiple Papio baboons were generated through the Baboon Genome Analysis Consortium and made available on NCBI. From this dataset we selected a diversity panel consisting of 12 Papio baboons: two from each of the six extant species. We then used our computational pipeline to process these WGS samples, targeting $A l u$ insertions present in multiple diversity samples, yet absent from the latest $M$. mulatta reference assembly [Mmul8.0.1]. In total, we identified 187,379 Alu insertions fitting this criterion.

\section{Basal divergence modeling}

We evaluated 31 distinct basal divergence models (see Methods), to determine the one best supported by our 
computational genotype data (Additional file 3: Figure S1). The model with the largest $\mathrm{z}$-score divided the Papio genus into two lineages: a northern clade containing $P$. papio, P. anubis, and P. hamadryas; and a southern clade consisting of $P$. cynocephalus, $P$. ursinus, and $P$. kindae (Additional file 3: Table S1). Of the 187,379 non-reference insertions (not present in Mmul8.0.1) reported in the previous section, 123,120 were concordant with this north-south basal divergence model $(\sim 66 \%)$ and 64,259 $(\sim 34 \%)$ were discordant.

\section{Papio phylogeny}

Using the data obtained from the panel of 12 Papio individuals, we constructed an Alu-based phylogeny of Papio baboons. For this analysis we used genotype data for 123,120 Alu insertions concordant with the north-south split hypothesis. The resulting cladogram resolved relationships within Papio with $100 \%$ bootstrap support at each node (C.I. $=0.703$, H.I. $=0.297)($ Fig. 2). Bootstrap values along with the total number of insertions supporting each node are included in Fig. 2.

To further examine evolutionary relationships within Papio, Alu insertions shared among multiple samples were clustered according to the patterns of shared Alu insertion polymorphisms determined for our Papio samples. This analysis was conducted multiple times, using various

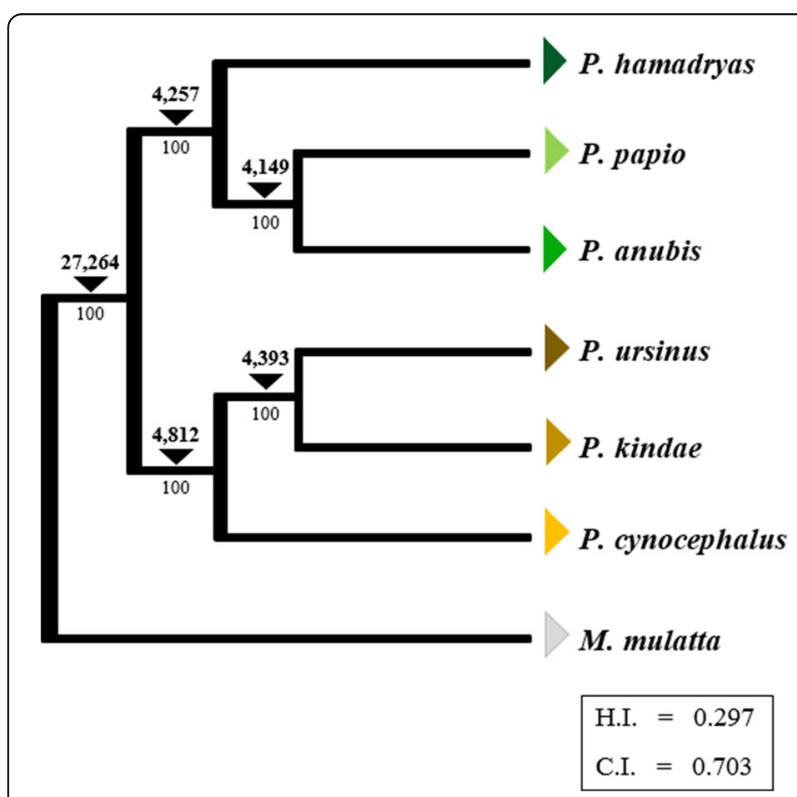

Fig. 2 Alu-based phylogeny of extant Papio baboon species. Phylogenetic relationships of Papio baboons constructed using 123,120 Alu insertion polymorphisms. Genotypes computationally determined in 12 Papio baboons were used to construct a Dollo parsimony tree using $M$. mulatta as an outgroup. The percentage of bootstrap replicates (out of 10,000 iterations) is listed below each branch; the number of Alu insertions supporting each node is listed above each branch. Homoplasy index (H.I.) and consistency index (C.I.) are included below the cladogram combinations of individuals from each species. Regardless of the representative individual selected for each species, the rank and size of each cluster, remained consistent. However, because we were particularly interested in observing clusters formed between individuals belonging to different species, we used one representative sample from each species. In each species, we selected the individual with sequencing coverage closest to the average coverage determined across all samples (Additional file 1). The resulting clusters are displayed in Fig. 3. Of the 187,379 Alu insertions identified in all 12 samples, we retained only those shared among multiple individuals from our panel of six Papio individuals. In total, we identified 106,204 such elements grouped into 57 unique clusters (For the full table, see Additional file 4). Figure 3 displays the 15 largest clusters, representing a total of 76,264 Alu insertions ( 72\% of the dataset). The largest cluster contained 32,156 markers present in all six Papio species (Fig. 3). Seven of the eight next largest clusters were shared exclusively between baboons belonging to the same clade (north/south). In total, these seven clusters contained 27,314 Alu insertions ( $26 \%$ of the dataset). Of the remaining clusters, four consisted of markers shared between five of the six Papio species (10,568 Alu insertions, $10 \%$ of the dataset), and three clusters consisted of insertions shared between $P$. kindae, and at least one of the northern baboons ( $6226 \mathrm{Alu}$ insertions, $\sim 6 \%$ of the dataset).

Northern and southern clade phylogenies were then re-evaluated using all 12 Papio baboons: two from each of the six extant Papio species, with all 187,379 Alu insertions. Alu insertions shared exclusively between multiple individuals belonging to the same clade were classified as clade-specific markers. A total of 95,703 such markers were identified: 39,795 in the northern clade and 55,908 in the southern clade. These markers were clustered based on precise presence/absence genotypes determined for all 12 Papio baboons. Species indicative markers were defined as $A l u$ insertions present in both individuals representing the same species, yet absent from all other members on the panel. In total we identified 48,808 species indicative markers: 23,578 markers were identified in the northern clade, with 25,230 identified in the southern clade. The total number of species indicative markers determined for each Papio species is displayed in Fig. 4a. Among northern baboon species, the highest number of species indicative Alu polymorphisms was determined for $P$. papio $(10,873)$, followed by $P$. hamadryas (8060) and $P$. anubis (4645). In the southern clade, $P$. kindae reported the highest number of species indicative markers $(12,891)$, followed by $P$. ursinus (9545), and $P$. cynocephalus (2794). Furthermore we evaluated inter-species relationships by targeting clade-specific markers shared between all individuals belonging to two species within a clade, yet absent from both individuals from the remaining 

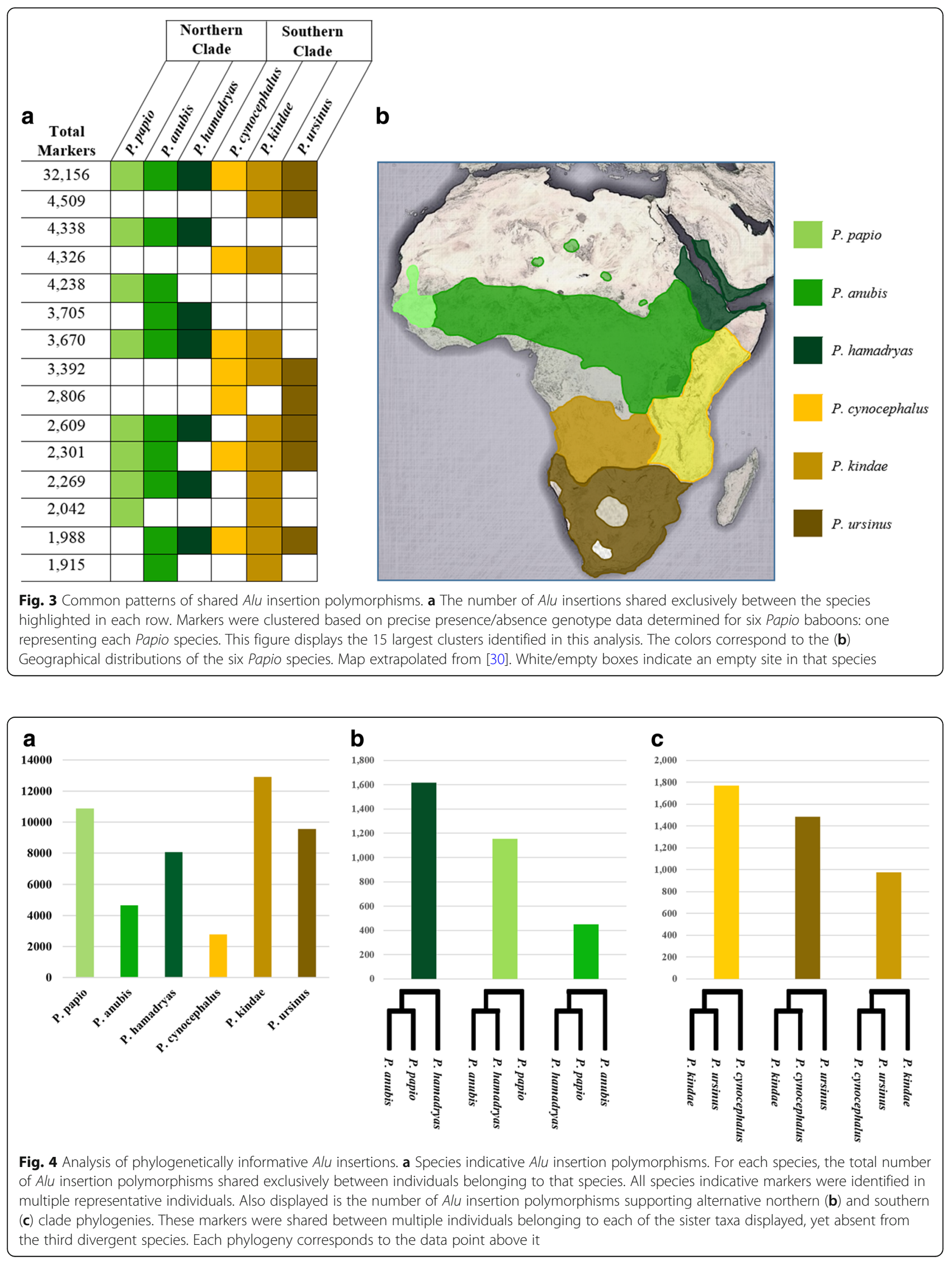
species. Within both clades, three unique clusters were formed from these data, each supporting a different clade phylogeny (Fig. 4b and c). A total of 7436 such elements was determined: 4220 in the northern clade and 3216 in the southern clade. Of the markers identified in the northern clade, $52 \%$ were shared exclusively between $P$. anubis and P. papio (1613 loci), 34\% were shared between $P$. anubis and P. hamadryas (1153 loci), and the remaining $14 \%$ were shared between $P$. papio and $P$. hamadryas (450 loci). In the southern clade analysis, 43\% of the Alu insertions were shared between P. ursinus and $P$. kindae (1766 loci), 36\% were shared between $P$. cynocephalus and $P$. ursinus (1483 loci), and $28 \%$ were shared between $P$. kindae and $P$. cynocephalus (971 loci).

In addition, we evaluated low-allele frequency Alu polymorphisms using data obtained from our complete panel of 12 individuals: two representing each Papio species. Alu insertions used in this analysis were those shared uniquely between only two species, and absent from the other four. Thus the overall number of these insertions among Papio was relatively low. We clustered these elements based on their precise presence/absence genotypes. Clusters identified for each species are displayed in Fig. 5. The numbers of insertions listed corresponds to the average of the two individuals from each species. With the exception of P. papio and $P$. hamadryas, the largest clusters identified in Papio species contained $A l u$ insertions shared between individuals belonging to the same clade (north/south). Although the single largest cluster identified in both $P$. papio and $P$. hamadryas consisted of elements shared with $P$. anubis, the second largest cluster was shared with $P$. kindae. All of the northern baboons shared more insertions with $P$. kindae than with the other two southern baboon species combined (P. cynocephalus and P. ursinus).

\section{Discussion}

With the increasing availability of WGS data, admixture remains a fundamental challenge for evolutionary biologists. Nevertheless, the abundance of genomic data provides scientists the opportunity to use novel methodologies to reexamine complex evolutionary relationships. Welldocumented extant hybrid zones coupled with a dense history of reticulation complicate the task of neatly organizing Papio baboons into a phylogenetic tree. Baboons are popular well-established research models for studying human disease and evolution, and therefore understanding the pattern of genetic variation within and between baboon species is important. As a result, an accurate and detailed understanding of Papio genomic evolution is quite valuable.

Despite the increasing availability of WGS data, high quality assemblies are not commonly constructed for multiple species belonging to the same genus. Instead, one individual is often used to build an assembly representative of an entire genus. However, often times WGS data are generated from individuals belonging to different species within that genus. For Papio baboons, a high quality (chromosome-level resolution) reference assembly exists only for Papio anubis, yet WGS data have been generated for

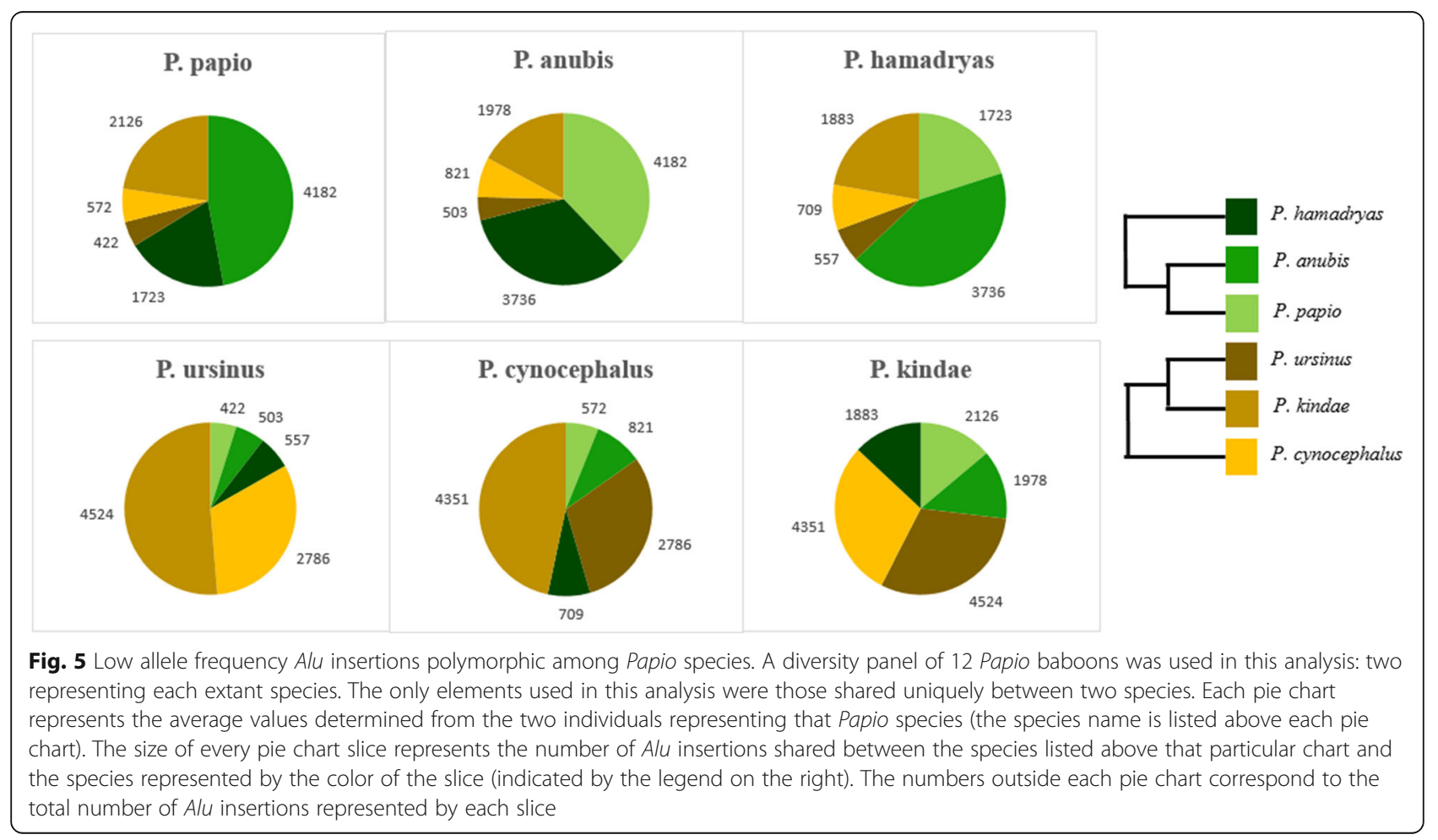


multiple individuals from each extant Papio species. A traditional method used to identify Alu elements polymorphic within a genus involved identifying markers present in an assembly of interest, yet absent from the closest primate relative with a draft assembly. For Papio baboons, a lineage-specific Alu polymorphism would be defined as an element present in P. anubis, yet absent in rhesus macaques [as represented by the assembly Mmul8.0.1]. Since all of the subsequent markers would be identified in a $P$. anubis individual, this would introduce sampling bias towards markers present in P. anubis. However, our computational approach allowed us to align all of our representative Papio samples against the outgroup rhesus macaque [Mmul8.0.1], placing equal evolutionary distance between each Papio individual and the reference assembly. As a result, we were able to identify polymorphic Alu elements with minimal directional bias.

Analyses conducted using mitochondrial DNA support the most basal divergence of Papio into northern and southern clades. However, these analyses were unable to produce a phylogeny that fully resolved evolutionary relationships between Papio species. Our findings provide support for this basal north-south split hypothesis. Furthermore, this study produces the first whole genome computational analysis of Alu polymorphisms within Papio. By designing a computational method to detect and characterize Alu polymorphisms from multiple Papio individuals representing all known extant species and evaluating various basal divergence models, we were able to produce a fully resolved phylogeny of Papio baboons with $100 \%$ bootstrap support at each node.

In addition, our analysis of elements discordant with this phylogenetic model may offer insights into a complex history of admixture and reticulation within the Papio lineage. In the southern lineage, $P$. kindae shows the highest incidence of $A l u$ insertions shared with the northern clade, yet absent from the other southern clade samples (11,286 elements). In total, we identified 64,259 elements discordant with topology of the phylogenetic tree (Fig. 2) that could be due to incomplete lineage sorting (ILS) or hybridization/admixture. Continued analyses involving a greater number of individuals would be necessary to accurately explain the taxonomic distribution of these insertions. Such analyses could potentially elucidate insertions indicative of speciation, the north-south split, hybridization, and many other evolutionary events. Thus, the data presented in this paper may be utilized to further evaluate Papio evolution. Such studies are likely necessary given the rich diversity that exists within the genus Papio. Furthermore, this approach has outstanding potential to inform analyses of other primate genera with complex evolutionary histories (e.g. Cercopithecus, Macaca, Chlorocebus, Aotus, Microcebus, Saimiri and others).

Contemporary arguments in favor of applying a phylogenetic species concept to the Papio genus rely heavily on the rich species diversity exhibited between morphotypes. Our findings provide support for the genetic diversity that exists within the genus Papio. In each extant species, we found an average of over 8000 elements shared exclusively between members belonging to that species. Despite previous debate as to whether $P$. kindae warrants species level classification, the largest number of species-specific elements characterized in this study were identified in P. kindae $(12,891)$.

One limitation of this study is that it is based on only 12 Papio individuals: two representing each species. It is very likely that the genetic diversity observed in each individual does not comprehensively represent diversity existing within the species as a whole. Each wild Papio species occupies a large range across the African continent; thus proximity to hybrid zones may contribute to interspecies diversity that is not captured in this analysis. Several species occupy ranges that contact other Papio species (Fig. 3b). Little is known about within species diversity. Only through further large-scale sampling and analyses can this be evaluated.

\section{Conclusions}

In conclusion, this study exhibits the utility and efficacy of a whole genome analysis of Alu polymorphisms for resolving controversial phylogenetic relationships. In addition, it demonstrates the importance of employing diverse methodologies. Knowledge of the initial divergence of Papio into northern and southern clades, produced by previous studies and supported in this study, was instrumental in our analysis of Papio evolution. Despite high incidence of hybridization and sustained hybrid zones, we were able to produce a highly supported cladogram, resolving relationships within both the northern and southern clades. These data represent the most comprehensive Alu-based phylogenetic reconstruction reported to date. In addition, this study also produces the first fully resolved Alu-based phylogeny of Papio baboons. Our approach may offer useful applications for investigating other unresolved branches of the primate evolutionary tree.

\section{Additional files}

\footnotetext{
Additional file 1: Sequencing information for the 13 WGS samples used in this study. Individuals listed in bold indicate the panel of Papio samples used to conduct the clustering analysis in which one representative sample was used for each species. All of the links provided in this file were last accessed March 2018. (XLSX 12 kb)

Additional file 2: An outline detailing the programs utilized in the computational pipeline. Command line arguments used in each run are provided. (DOCX $14 \mathrm{~kb}$ )

Additional file 3: An excel file containing a figure and table representing possible basal divergence model reconstructions generated using all six extant Papio species. A maximum of 31 rooted monophyletic models can be generated from such a genus comprised of six species. These models can be further organized into three distinct groups based on the number of
} 
species contained in the subsequent clades. Group I depicts the six different scenarios when one of the six species diverges prior to the other five. Group I-A) illustrates P. kindae diverging first, followed by B) P. ursinus first, then C-F) P. cynocephalus, P. papio, P. hamadryas, and P. anubis diverging first, respectively. Group II depicts the 15 different models when two of the six species diverge prior to the other four. All possible combinations of this scenario are illustrated in Group II A-O. Group III depicts the ten different models generated from a basal divergence that forms two clades each comprised of three species. All ten combinations are listed in Group III A-J. The values listed correspond to the 31 possible phylogenetic models displayed in Figure S1. For each model, the number of concordant insertions are provided in the third column; the number of discordant insertions can be found in the fourth column. The $z$-score determined for the number of discordant insertions is listed in the last column. The lowest z-score (indicating smallest proportion of discordant elements in group) is shown in bold font and indicates scenario III-A to be the most likely basal divergence model. (DOCX $61 \mathrm{~kb}$ )

Additional file 4: An extension of Fig. 3. It is an excel file containing the complete cluster list: all 57 clusters identified in this analysis. (XLSX $13 \mathrm{~kb}$ )

Additional file 5: This file lists the members of the Baboon Genome Analysis Consortium as well as their contact information. (DOCX $13 \mathrm{~kb}$ )

\section{Abbreviations}

LINE: Long interspersed element; LTR: Long terminal repeat; SINE: Short interspersed element; TPRT: Target-primed reverse transcription; WGS: Whole genome sequencing

\section{Acknowledgements}

The authors would like to thank all the members of the Batzer Lab and the Baboon Genome Analysis Consortium for all of their hard work and thoughtful insights.

Membership in the Baboon Genome Analysis Consortium is listed as Additional file 5.

\section{Funding}

This research was funded by the National Institutes of Health R01 GM59290 (M.A.B.).

\section{Availability of data and materials}

The algorithms used in this study are available on GitHub (https://github.com/ papioPhlo/polyDetect; last accessed March 2018). The Additional Information files are available on the online version of this paper and through the Batzer Lab website under publications, https://biosci-batzerlab.biology.lsu.edu; last accessed March 2018. Additional file 1, A1, is an excel document (.docx) containing a WGS samples list. This file provides information for all of the WGS samples used in this analysis including a link to the WGS data publicly available on the NCBI-SRA database. Additional file 2, A2, is a word document (.docx) providing a more detailed description of the computational pipeline. Additional file $3, \mathrm{~A} 3$, is a word document (.docx) providing all of the possible basal divergence models with the corresponding statistics determine for each. All datasets generated or analyzed in this study will be available from the corresponding author upon reasonable request. Additional file 4, A4, is an extension of Fig. 3. It is an excel file (.docx) containing the complete cluster list: all 57 clusters identified in this analysis. Additional file 5, A5, is a word document (.docx) containing Baboon Genome Analysis Consortium Member Information. This file lists the members of the Baboon Genome Analysis Consortium as well as their contact information.

\section{Authors' contributions}

VEJ, JAW, KCW, CJJ, JR, MKK, and MAB designed the research. VEJ and TOB designed the computational algorithm and performed computational analyses. VEJ, JAW, CJS, TOB, CLM, and CPS-R conducted the PCR validation experiments. VEJ wrote the manuscript; JAW, KCW, JP-C, CJJ, JR, MKK, and $M A B$ revised the manuscript. All authors read and approved the final manuscript.

\section{Ethics approval and consent to participate}

Not applicable.

\section{Consent for publication}

Not applicable.

\section{Competing interests}

The authors declare that they have no competing interests.

\section{Publisher's Note}

Springer Nature remains neutral with regard to jurisdictional claims in published maps and institutional affiliations.

\section{Author details}

1Department of Biological Sciences, Louisiana State University, 202 Life Sciences Building, Baton Rouge, LA 70803, USA. ${ }^{2}$ Human Genome Sequencing Center, Baylor College of Medicine, Houston, TX 77030, USA. ${ }^{3}$ Department of Molecular and Human Genetics, Baylor College of Medicine, Houston, TX 77030, USA. ${ }^{4}$ Department of Neuroscience, Washington University School of Medicine, St. Louis, MO 63110, USA. ${ }^{5}$ Department of Anthropology, New York University, New York, NY 10003, USA. ${ }^{6}$ Department of Genetics \& Biochemistry, Clemson University, Clemson, SC 29634, USA.

Received: 30 November 2017 Accepted: 26 March 2018

Published online: 05 April 2018

\section{References}

1. Bontrop RE. Non-human primates: essential partners in biomedical research. Immunol Rev. 2001;183(1):5-9.

2. Jolly CJ. A proper study for mankind: analogies from the Papionin monkeys and their implications for human evolution. Am J Phys Anthropol. 2001; 116(S33):177-204.

3. Patterson JL, Carrion R. Demand for nonhuman primate resources in the age of biodefense. ILAR J. 2005;46(1):15-22.

4. Goodman S, Check E. Animal experiments: the great primate debate. Nature. 2002:417(6890):684-7.

5. Phillips KA, Bales KL, Capitanio JP, Conley A, Czoty PW, Hart BA, Hopkins WD, Hu S-L, Miller LA, Nader MA, et al. Why primate models matter. Am J Primatol. 2014;76(9):801-27.

6. Cox LA, Comuzzie AG, Havill LM, Karere GM, Spradling KD, Mahaney MC, Nathanielsz PW, Nicolella DP, Shade RE, Voruganti S, et al. Baboons as a model to study genetics and epigenetics of human disease. ILAR J. 2013; 54(2):106-21.

7. Dormehl IC, Hugo N, Beverley G. The baboon: an ideal model in biomedical research. Anesth Pain Control Dent. 1992;1(2):109-15.

8. VandeBerg JL, Williams-Blangero S, Tardif SD. The baboon in biomedical research: Springer New York; 2009.

9. McGill HC, McMahan CA, Kruski AW, Mott GE. Relationship of lipoprotein cholesterol concentrations to experimental atherosclerosis in baboons. Arterioscler Thromb Vasc Biol. 1981;1(1):3-12.

10. Jerome C, Kimmel D, McAlister J, Weaver D. Effects of ovariectomy on iliac trabecular bone in baboons (Papio anubis). Calcif Tissue Int. 1986;39(3):206-8.

11. Kammerer CM, Cox LA, Mahaney MC, Rogers J, Shade RE. Sodium-lithium countertransport activity is linked to chromosome 5 in baboons. Hypertension. 2001;37(2):398-402.

12. Strum SC, Mitchell W. Baboon models and muddles. In: Kinzey WG, editor The evolution of human behavior: primate models. New York: New York State University Press; 1987. p. 87-104.

13. Rodseth L, Wrangham RW, Harrigan AM, Smuts BB, Dare R, Fox R, King BJ, Lee PC, Foley RA, Muller JC, et al. The human community as a primate society [and comments]. Curr Anthropol. 1991:32(3):221-54.

14. Jablonski NG, Frost SR. Cercopithecoidea. In: Cenozoic mammals of Africa. Werdelin L, Sanders WJ. Berkeley: University of California Press; 2010. p. 393-428.

15. Racimo F, Sankararaman S, Nielsen R, Huerta-Sanchez E. Evidence for archaic adaptive introgression in humans. Nat Rev Genet. 2015;16(6):359-71.

16. Sankararaman S, Mallick S, Dannemann M, Prüfer K, Kelso J, Pääbo S, Patterson N, Reich D. The landscape of Neandertal ancestry in present-day humans. Nature. 2014;507(7492):354-7.

17. Kopp GH, Roos C, Butynski TM, Wildman DE, Alagaili AN, Groeneveld LF, Zinner D. Out of Africa, but how and when? The case of hamadryas baboons (Papio hamadryas). J Hum Evol. 2014;76:154-64

18. Winney BJ, Hammond RL, Macasero W, Flores B, Boug A, Biquand V, Biquand S, Bruford MW. Crossing the Red Sea: phylogeography of the hamadryas baboon, Papio hamadryas hamadryas. Mol Ecol. 2004;13(9):2819-27.

19. Groucutt HS, Petraglia MD, Bailey G, Scerri EML, Parton A, Clark-Balzan L, Jennings RP, Lewis L, Blinkhorn J, Drake NA, et al. Rethinking the dispersal of Homo sapiens out of Africa. Evol Anthropol. 2015;24(4):149-64. 
20. Wildman DE, Bergman TJ, al-Aghbari A, Sterner KN, Newman T, PhillipsConroy JE, Jolly CJ, Disotell TR. Mitochondrial evidence for the origin of hamadryas baboons. Mol Phylogenet Evol. 2004;32:287.

21. Barrett L, Henzi SP. Baboons. Curr Biol. 2008;18(10):R404-6.

22. Caldecott JO, Miles L. Programme UNE, Centre WCM: world atlas of great apes and their conservation: University of California Press; 2005.

23. Eeley HAC, Foley R. Species richness, species range size and ecological specialisation among African primates: geographical patterns and conservation implications. Biodivers Conserv. 1999;8(8):1033-56.

24. Jolly CJ. Species, subspecies, and baboon systematics. In: Kimbel WH, Martin LB, editors. Species, species concepts and primate evolution. Boston: Springer US; 1993. p. 67-107.

25. Groves CP. Primate taxonomy. Washington DC: Smithsonian Institution Press; 2001.

26. Zinner D, Wertheimer J, Liedigk R, Groeneveld LF, Roos C. Baboon phylogeny as inferred from complete mitochondrial genomes. Am J Phys Anthropol. 2013;150(1):133-40.

27. Jolly CJ, Burrell AS, Phillips-Conroy JE, Bergey C, Rogers J. Kinda baboons (Papio kindae) and grayfoot chacma baboons (P. ursinus griseipes) hybridize in the Kafue river valley, Zambia. Am J Primatol. 2011;73(3):291-303.

28. Walker JA, Jordan VE, Steely CJ, Beckstrom TO, McDaniel CL, St. Romain CP, Bennett EC, Robichaux A, Clement BN, Konkel MK, et al. Papio baboon species indicative Alu elements. Genome Biol Evol. 2017;9(6):1788-96.

29. Steely CJ, Walker JA, Jordan VE, Beckstrom TO, McDaniel CL, St Romain CP, Bennett EC, Robichaux A, Clement BN, Raveendran M, et al. Alu insertion polymorphisms as evidence for population structure in baboons. Genome Biol Evol. 2017;9(9):2418-27.

30. Zinner D, Groeneveld LF, Keller C, Roos C. Mitochondrial phylogeography of baboons (Papio spp.) - indication for introgressive hybridization? BMC Evol Biol. 2009;9:83.

31. Alberts SC, Altmann J. Immigration and hybridization patterns of yellow and anubis baboons in and around Amboseli, Kenya. Am J Primatol. 2001;53:139.

32. Bergman T, Phillips-Conroy JE, Jolly CJ. Behavioral variation and reproductive success of male baboons (Papio anubis $\times$ Papio hamadryas) in a hybrid social group. Am J Primatol. 2008;70:136.

33. Maples WR, McKern TW. A preliminary report on classification of the Kenya baboon. In: Vagtborg H, editor. The baboon in medical research, vol. 2. San Antonio: University of Texas Press; 1967. p. 13-22.

34. Phillips-Conroy JE, Jolly CJ, Brett FL. Characteristics of hamadryas-like male baboons living in anubis baboon troops in the Awash hybrid zone, Ethiopia. Am J Phys Anthropol. 1991;86(3):353-68.

35. Nagel U. A comparison of anubis baboons, hamadryas baboons and their hybrids at a species border in Ethiopia. Folia Primatol. 1973;19:104

36. Szmulewicz MN, Andino LM, Reategui EP, Woolley-Barker T, Jolly CJ, Disotell TR, Herrera RJ. An Alu insertion polymorphism in a baboon hybrid zone. Am J Phys Anthropol. 1999;109(1):1-8.

37. McLain AT, Meyer TJ, Faulk C, Herke SW, Oldenburg JM, Bourgeois MG, Abshire CF, Roos C, Batzer MA. An Alu-based phylogeny of lemurs (Infraorder: Lemuriformes). PLoS One. 2012;7(8):e44035.

38. Salem A-H, Ray DA, Xing J, Callinan PA, Myers JS, Hedges DJ, Garber RK, Witherspoon DJ, Jorde LB, Batzer MA. Alu elements and hominid phylogenetics. Proc Natl Acad Sci. 2003;100(22):12787-91.

39. Meyer TJ, McLain AT, Oldenburg JM, Faulk C, Bourgeois MG, Conlin EM, Mootnick AR, de Jong PJ, Roos C, Carbone L, et al. An Alu-based phylogeny of gibbons (Hylobatidae). Mol Biol Evol. 2012;29(11):3441-50.

40. Watkins WS, Rogers AR, Ostler CT, Wooding S, Bamshad MJ, Brassington AM, Carroll ML, Nguyen SV, Walker JA, Prasad BV, et al. Genetic variation among world populations: inferences from 100 Alu insertion polymorphisms. Genome Res. 2003;13(7):1607-18.

41. Xing J, Wang H, Han K, Ray DA, Huang CH, Chemnick LG, Stewart C-B, Disotell TR, Ryder OA, Batzer MA. A mobile element based phylogeny of Old World monkeys. Mol Phylogenet Evol. 2005;37(3):872-80.

42. Li J, Han K, Xing J, Kim H-S, Rogers J, Ryder OA, Disotell T, Yue B, Batzer MA. Phylogeny of the macaques (Cercopithecidae: Macaca) based on Alu elements. Gene. 2009;448(2):242-9.

43. Schmitz J, Ohme M, Zischler H. SINE insertions in cladistic analyses and the phylogenetic affiliations of <em $>$ Tarsius bancanus $</$ em $>$ to other primates. Genetics. 2001;157(2):777-84.

44. Xing J, Wang H, Zhang Y, Ray DA, Tosi AJ, Disotell TR, Batzer MA. A mobile element-based evolutionary history of guenons (tribe Cercopithecini). BMC Biol. 2007;5(1):5
45. Shedlock AM, Okada N. SINE insertions: powerful tools for molecular systematics. BioEssays. 2000;22:148.

46. Batzer MA, Arcot SS, Phinney JW, Alegria-Hartman M, Kass DH, Milligan SM, Kimpton C, Gill P, Hochmeister M, loannou PA, et al. Genetic variation of recent Alu insertions in human populations. J Mol Evol. 1996;42(1):22-9.

47. Stoneking M, Fontius JJ, Clifford SL, Soodyall H, Arcot SS, Saha N, Jenkins T, Tahir MA, Deininger PL, Batzer MA. Alu insertion polymorphisms and human evolution: evidence for a larger population size in Africa. Genome Res. 1997;7(11):1061-71.

48. Batzer MA, Deininger PL. Alu repeats and human genomic diversity. Nat Rev Genet. 2002;3(5):370-9.

49. Deininger PL, Batzer MA. Mammalian retroelements. Genome Res. 2002; 12(10):1455-65.

50. Roy-Engel A, Batzer MA, Deininger P. Evolution of human retrosequences: Alu; 2008.

51. Lander ES, Linton LM, Birren B, Nusbaum C, Zody MC, Baldwin J, Devon K, Dewar K, Doyle M, FitzHugh W. Initial sequencing and analysis of the human genome. Nature. 2001;409:860.

52. Chimpanzee Sequencing and Analysis Consortium. Initial sequence of the chimpanzee genome and comparison with the human genome. Nature. 2005:437(7055):69-87.

53. Gibbs RA, Rogers J, Katze MG, Bumgarner R, Weinstock GM, Mardis ER, Remington KA, Strausberg RL, Venter JC, Wilson RK, et al. Evolutionary and biomedical insights from the rhesus macaque genome. Science. 2007; 316(5822):222-34

54. Locke DP, Hillier LW, Warren WC, Worley KC, Nazareth LV, Muzny DM, Yang SP, Wang Z, Chinwalla AT, Minx P, et al. Comparative and demographic analysis of orang-utan genomes. Nature. 2011;469(7331):529-33.

55. Scally A, Dutheil JY, Hillier LW, Jordan GE, Goodhead I, Herrero J, Hobolth A, Lappalainen T, Mailund T, Marques-Bonet T, et al. Insights into hominid evolution from the gorilla genome sequence. Nature. 2012;483:169.

56. Luan DD, Korman MH, Jakubczak JL, Eickbush TH. Reverse transcription of R2Bm RNA is primed by a nick at the chromosomal target site: a mechanism for non-LTR retrotransposition. Cell. 1993;72(4):595-605.

57. Luan DD, Eickbush TH. RNA template requirements for target DNA-primed reverse transcription by the $\mathrm{R} 2$ retrotransposable element. Mol Cell Biol. 1995;15(7):3882-91.

58. Cost GJ, Feng Q, Jacquier A, Boeke JD. Human L1 element target-primed reverse transcription in vitro. EMBO J. 2002;21(21):5899-910.

59. Comeaux MS, Roy-Engel AM, Hedges DJ, Deininger PL. Diverse cis factors controlling Alu retrotransposition: what causes Alu elements to die? Genome Res. 2009;19(4):545-55.

60. Dewannieux M, Esnault C, Heidmann T. LINE-mediated retrotransposition of marked Alu sequences. Nat Genet. 2003;35(1):41-8.

61. Ray DA, Xing J, Salem AH, Batzer MA. SINEs of a nearly perfect character. Syst Biol. 2006;55:928.

62. Konkel MK, Walker JA, Batzer MA. LINEs and SINEs of primate evolution. Evol Anthropol. 2010;19(6):236-49.

63. Batzer MA, Deininger PL. A human-specific subfamily of Alu sequences. Genomics. 1991;9:481-7.

64. Roos C, Geissmann T. Molecular phylogeny of the major hylobatid divisions Mol Phylogenet Evol. 2001;19(3):486-94.

65. Coordinators NR. Database resources of the national center for biotechnology information. Nucleic Acids Res. 2016;44(Database issue):D7-d19.

66. Leinonen $\mathrm{R}$, Sugawara $\mathrm{H}$, Shumway $\mathrm{M}$, on behalf of the International Nucleotide Sequence Database C. The sequence read archive. Nucleic Acids Res. 2011;39(Database issue):D19-21.

67. Bennett EA, Keller H, Mills RE, Schmidt S, Moran JV, Weichenrieder O, Devine SE. Active Alu retrotransposons in the human genome. Genome Res. 2008; 18(12):1875-83.

68. Batzer MA, Kilroy GE, Richard PE, Shaikh TH, Desselle TD, Hoppens CL, Deininger PL. Structure and variability of recently inserted Alu family members. Nucleic Acids Res. 1990;18(23):6793-8.

69. Carroll ML, Roy-Engel AM, Nguyen SV, Salem A-H, Vogel E, Vincent B, Myers J, Ahmad Z, Nguyen L, Sammarco M, et al. Large-scale analysis of the Alu Ya5 and Yb8 subfamilies and their contribution to human genomic diversity11Edited by J. Karn. J Mol Biol. 2001;311(1):17-40.

70. Li H. Aligning sequence reads, clone sequences and assembly contigs with BWA-MEM, vol. 1303; 2013.

71. Jurka J. Repeats in genomic DNA: mining and meaning. Curr Opin Struct Biol. 1998;8(3):333-7. 
72. Langmead B, Salzberg SL. Fast gapped-read alignment with bowtie 2. Nat Methods. 2012;9(4):357-9.

73. Swofford DL. PAUP*: phylogenetic analysis using parsimony, version 4. Ob10. 2011.

74. Durrant SD, Simpson GG. The major features of evolution. J Mammal. 1953; 35(4):600-1.

Submit your next manuscript to BioMed Central and we will help you at every step:

- We accept pre-submission inquiries

- Our selector tool helps you to find the most relevant journal

- We provide round the clock customer support

- Convenient online submission

- Thorough peer review

- Inclusion in PubMed and all major indexing services

- Maximum visibility for your research

Submit your manuscript at www.biomedcentral.com/submit 